\title{
MARCO DE REFERENCIA PARA LA GESTIÓN DE PROGRAMAS EN E-SALUD
}

\author{
Arturo Serrano-Santoyo ${ }^{1}$, Verónica A. Rojas-Mendizábal², \\ Cristián Castillo-Olea ${ }^{3}$
}
${ }^{1}$ Doctor en Ciencias en Ingeniería Eléctrica. Investigador, Centro de Investigación Científica y Educación Superior de Ensenada; profesor de tiempo completo, CETYS Universidad, Ensenada, Baja California, México. Correo electrónico: arturo.serrano@cetys.mx
${ }^{2}$ Doctora en Ciencias, Centro de Investigación Científica y Educación Superior de Ensenada, Ensenada, México
${ }^{3}$ Doctora en Estudios de Desarrollo Global. Investigadora, Facultad de Ingeniería, Universidad de Deusto, Bilbao, Vizcaya, España

Resumen: Introducción: producto de la investigación "Innovación, complejidad y gestión en sistemas sociotécnicos", desarrollada entre 2014-2017 en el Centro de Investigación Científica y Educación Superior de Ensenada, Baja California, México. Los sistemas de procesamiento, distribución y transporte de información digital tienen hoy gran impacto en la operación del sector salud, creando oportunidades para mejorar sus servicios, cobertura y los procesos de información sanitaria de carácter administrativo, clínico y operativo. Una perspectiva de la salud electrónica o salud digital (e-salud) desde la ciencia de la complejidad puede extraer nuevos hallazgos hacia el entendimiento de su ecosistema en los ámbitos local, regional y nacional, lo que aumenta su efectividad como potencial habilitador de bienestar social. Metodología: se utilizó la ciencia de la complejidad para desarrollar un marco de referencia, teniendo en cuenta las interacciones entre los agentes del ecosistema y las características del contexto donde los programas e intervenciones de e-salud serán implantados. Resultados: se presenta un marco de referencia para abordar programas, proyectos e intervenciones de e-salud desde una perspectiva integral; se tiene en cuenta el papel que juegan las interacciones de los agentes preponderantes del sistema y las características del contexto que se intervendrá. Como ejemplo de aplicación del marco de referencia propuesto, se describe el planteamiento de proyecto de "Teleepidemiología de enfermedades transmitidas por vector" en proceso de desarrollo por nuestro grupo de investigación. Conclusiones: el marco de referencia propuesto provee una plataforma de análisis y gestión que intenta aprovechar el potencial de la e-salud para convertirse en vehículo efectivo de solidaridad y desarrollo social.

Palabras clave: ecosistemas, innovación, políticas públicas, sistemas complejos, telesalud. 


\section{Frame OF REFERENCE MANAGING E-HEALTH PROGRAMS}

Abstract. Introduction: This article derives from the research "Innovation, complexity, and management in socio-technical systems". It was conducted between 2014 and 2017 at the Center for Scientific Research and Higher Education of Ensenada, Baja California, Mexico. The systems for processing, distributing, and transporting digital information currently have a great impact on the operation of the healthcare industry, creating opportunities to improve its services and coverage and seeking to streamline health information processes of an administrative, clinical and operational nature. A perspective of electronic health or digital health (e-health) from the science of complexity can extract new findings towards the understanding of the e-health ecosystem at the local, regional and national levels, increasing its effectiveness as a potential enabler of social welfare. Methodology: This article turns to the science of complexity as a methodological basis to develop a frame of reference, considering the interactions between agents of the ecosystem and the characteristics of the context where e-health programs and interventions will be implemented. Results: A frame of reference is presented to address e-health programs, projects and interventions from a comprehensive perspective taking into account the role played by the interactions between predominant agents of the system and the characteristics of the context that will be intervened. As an example of application of the proposed frame of reference, the project "Tele-epidemiology of vector-borne diseases" being carried out by our research group is briefly described. Conclusions: The proposed frame of reference provides a platform for analysis and management that seeks to take advantage of the potential of e-health to become an effective vehicle for solidarity and social development.

Keywords: ecosystems, innovation, public policies, complex systems, telehealth.

\section{MARCo de REFERÊNCIA PARA A GeSTÃo DE PROGRAMAS EM E-SAÚDE}

Resumo. Introdução: o artigo é produto da pesquisa "Inovação, complexidade e gestão em sistemas sociotécnicos", que foi desenvolvida entre 2014 e 2017 no Centro de Pesquisa Científica e Educação Superior de Ensenada, Baixa Califórnia, no México. Os sistemas de processamento, distribuição e transporte de informação digital têm atualmente grande impacto na operação do setor de saúde, o que cria oportunidades para melhorar seus serviços e cobertura e para tentar tornar eficientes os processos de informação sanitária de caráter administrativo, clínico e operativo. Uma perspectiva da saúde eletrônica ou saúde digital (e-saúde) desde a ciência da complexidade pode extrair novas descobertas para o entendimento do ecossistema da e-saúde nos âmbitos local, regional e nacional, o que aumenta sua efetividade como potencial habilitador de bem-estar social. Metodologia: neste artigo recorremos à ciência da complexidade como base metodológica para desenvolver um marco de referência levando em consideração as interações entre os agentes do ecossistema e as características do contexto no qual os programas e intervenções de e-saúde serão implantados. Resultados: apresenta-se um marco de referência para abordar os programas, projetos e intervenções de e-saúde desde uma perspectiva integral, levando em consideração o papel das interações dos agentes preponderantes do sistema e as características do contexto que passará por intervenção. Como exemplo de aplicação do marco de referência proposto, descreve-se brevemente a concepção do projeto de "Tele-epidemiologia de doenças transmitidas por vetor" em processo de desenvolvimento por nosso grupo de pesquisa. Conclusões: o marco de referência proposto fornece uma plataforma de análise e gestão que tenta aproveitar o potencial da e-saúde para se converter em um veículo efetivo de solidariedade e desenvolvimento social.

Palavras-chave: ecossistemas, inovação, políticas públicas, sistemas complexos, telessaúde. 


\section{Introducción}

En el entorno de las aplicaciones de las tecnologías de la información y comunicación (TIC) en el sector salud, se utilizan diferentes términos relacionados entre sí. Según la Organización Mundial de la Salud (oms) [1], la telemedicina se define como el uso de las telecomunicaciones para diagnosticar y tratar enfermedades, incluyendo intervenciones clínicas. La telesalud incluye la vigilancia, la promoción de salud y el funcionamiento de salud pública; es un término más amplio que telemedicina e incluye la asistencia de computadoras y telecomunicaciones para el apoyo de gestión, vigilancia, literatura $\mathrm{y}$ acceso al conocimiento en medicina (teleeducación). E-salud es un término aún más amplio: aparte de considerar las funciones de telesalud y telemedicina, considera la transferencia de los recursos y cuidado de la salud para profesionales y usuarios por medios electrónicos, e incluye el uso de comercio electrónico en la práctica de salud.

En la e-salud intervienen elementos socioculturales que juegan un papel clave en la adopción y apropiación de la tecnología en el sector salud. El considerar la naturaleza multidimensional de la e-salud y la preponderancia del usuario final como su centro de gravedad nos permite argumentar que, para el entendimiento de la operación de dicho ecosistema de forma integral, no es suficiente una visión disciplinaria. La adopción de la convergencia tecnológica, el incentivar la creación y fortalecimiento de infraestructura, y, sobre todo, cuidar que la calidad y cobertura de los servicios de e-salud sean justos y asequibles a la población, requiere un nuevo enfoque. Este enfoque es imprescindible al observar la condición y potencial de las de las TIC como vehículo de prosperidad social.

En cuanto al estado del arte, en los avances recientes de e-salud convergen diferentes disciplinas tales como la genómica, la inteligencia artificial, la nano y biotecnología; de tal forma que nuestra propuesta de estudiar la e-salud desde una perspectiva integral puede dar pie a conocer a mayor profundidad las implicaciones socioeconómicas, regulatorias y normativas de las tecnologías que emergerán de la confluencia de los esfuerzos de innovación y desarrollo impulsados por el acelerado cambio tecnológico [2].

Nuestra propuesta parte de la lectura de la realidad del contexto para entender la dinámica del ecosistema de e-salud. Una intervención o proyecto de e-salud está sujeto a interacciones con agentes internos y externos que dan lugar al surgimiento de propiedades y comportamientos no esperados y a procesos de autoorganización y retroalimentación, atributos que denotan su naturaleza dinámica, adaptiva y compleja [3]-[6].

Se propone entonces que el entendimiento de la dinámica del ecosistema de e-salud permite plantear de manera integral los retos, objetivos y preguntas conductoras de proyectos y programas locales, regionales y nacionales. Lo anterior puede apoyar al diseño e implementación de políticas públicas que se apartan de enfoques reduccionistas que privilegian el "hacer más de lo mismo", en los cuales el otorgamiento de insumos (tecnológicos y financieros) no garantizan por sí solos la eficiencia de los proyectos.

El objetivo de este artículo es explorar el ecosistema de e-salud desde la perspectiva de la complejidad y ofrecer un marco conceptual para hacer una aproximación sistemática con énfasis en la formulación y propuestas de implementación de políticas públicas que, por un lado, contribuyan a un mejor planteamiento de los proyectos, programas e intervenciones de e-salud, y, por otro, promover la creación de contextos de interacción en continuo aprendizaje para mejorar servicios y procesos de e-salud [7].

El presente artículo está organizado de la siguiente manera: en la sección 2 se discute el proceso de construcción de la e-salud como un sistema dinámico complejo. En la sección 3 se presenta nuestra propuesta de gestión desde la perspectiva de la complejidad, con el fin de contribuir a la pertinencia de políticas públicas de acuerdo al contexto de intervención de la e-salud. En la sección 4 se presenta un resumen de una aplicación del marco de referencia propuesto para el caso de teleepidemiología. Finalmente, se presentan las conclusiones reconociendo el inicio de una búsqueda por lograr plantear de una manera holística e inclusiva los problemas relativos a proyectos de creación y fortalecimiento de infraestructura y procesos de e-salud. Lo anterior se hace mediante un enfoque interdisciplinario que atiende las necesidades de los actores del ecosistema con mediación de la tecnología para hacer eficientes sus funciones y lograr su propósito de bienestar social. 


\section{Metodología: el espacio de estudio de la e-salud bajo los principios de los sistemas complejos}

Los componentes, procesos e intervenciones de e-salud no pueden verse de manera aislada y en la actualidad no pueden ser conceptualizados solo como una infraestructura de equipos, por lo que un abordaje en forma fragmentada limita su efectividad e impacto social [8].

$\mathrm{Al}$ introducir la complejidad como propuesta de análisis de la e-salud, se conceptualiza el pensamiento complejo sobre este sistema sociotécnico como un corpus de ideas coherentemente conectadas que permiten observar un corte de la realidad del funcionamiento en el sistema, que ayuda, a su vez, a entender los cambios y las interrelaciones de los subsistemas y agentes involucrados [9].

Las características colectivas de un sistema complejo no pueden ser previstas en su totalidad a partir de sus componentes individuales. La interconectividad es la esencia de los sistemas complejos, es decir, estos sistemas comprenden una red de nodos interconectados cuyas interacciones definen su naturaleza [10]. Un entorno se "complejiza" cuando existen diferentes subsistemas que interactúan de tal forma que el total adquiere una naturaleza por sí mismo, se adapta y se desempeña en respuesta a condiciones cambiantes, y donde nuevas estructuras con nuevas propiedades surgen como resultado de la interacción de sus componentes [11].

En este artículo se propone que la e-salud constituye un sistema sociotécnico, en el cual la interacción e interdependencia de los elementos tecnológicos, socioculturales y económicos juegan un papel clave en su caracterización. Para llevar a cabo nuestro análisis acudimos a la ciencia de la complejidad, la cual ofrece una perspectiva que ayuda a entender cómo los contextos sociales, económicos y políticos se interrelacionan. Permite, a la vez, observar cómo los sistemas sociotécnicos, de los cuales las personas son parte, no son mecánicos, sino impredecibles, con procesos de retroalimentación, surgimiento (emergencia), interdependencia y autoorganización. Así mismo, una perspectiva compleja puede extraer nuevos hallazgos rumbo a la conformación de un marco de referencia que involucre a los actores de mayor relevancia, así como los diferentes niveles de gestión que intervienen en la dinámica del sistema [12], [13].

Por lo anteriormente expuesto, el comportamiento multifactorial de la e-salud y la interacción entre sus componentes admite su conceptualización como un sistema sociotécnico y, adicionalmente, complejo, dinámico y adaptivo, en el cual se hace necesario romper con patrones paradigmáticos que solo se enfocan en el lado tecnológico del sistema [11].

En la conjugación práctica de los anteriores planteamientos, se asume un acoplamiento entre los individuos y la tecnología, y entre los individuos con mediación de la tecnología mediante dispositivos informáticos para producir un intercambio de información, que da lugar a cambios de patrones y procesos en la sociedad y en el ecosistema de e-salud, y así cumplir con su propósito (figura 1). En esta perspectiva, las interacciones entre pacientes, especialistas médicos, administradores y tomadores de decisión del entorno socioeconómico y cultural (contexto) utilizan la tecnología como medio para llegar a diversos objetivos, como son ampliar la cobertura en zonas aisladas y remotas, educación e investigación, prevención, diagnóstico, rehabilitación, monitoreo, etc. [14].

De la misma forma, los aspectos de convergencia e interdisciplinarios adquieren relevancia en torno al trabajo colaborativo que la e-salud exige para su estudio y tratamiento integral. Se propone entonces que el abordaje de un problema o conjunto de problemas sobre e-salud se lleve a cabo mediante un proceso de resolución de problemas. Como Rolando García plantea: "para pensar diferente alrededor de la solución de un problema”. Es decir, la interdisciplinariedad se da por la forma en que se aborda el problema, no por el número o tipo de disciplinas que los miembros de un equipo de trabajo dominen, es decir, se requiere la coordinación de los enfoques disciplinarios para integrarlos en un enfoque común [13], [15]. En suma, la interdisciplinariedad se concibe, para propósitos de la presente exploración, como un proceso más que como un producto.

En el proceso de construcción de la e-salud como sistema complejo, adoptamos la propuesta de Rolando García [13] de un marco conceptual y metodológico de trabajo interdisciplinario. Se propone 


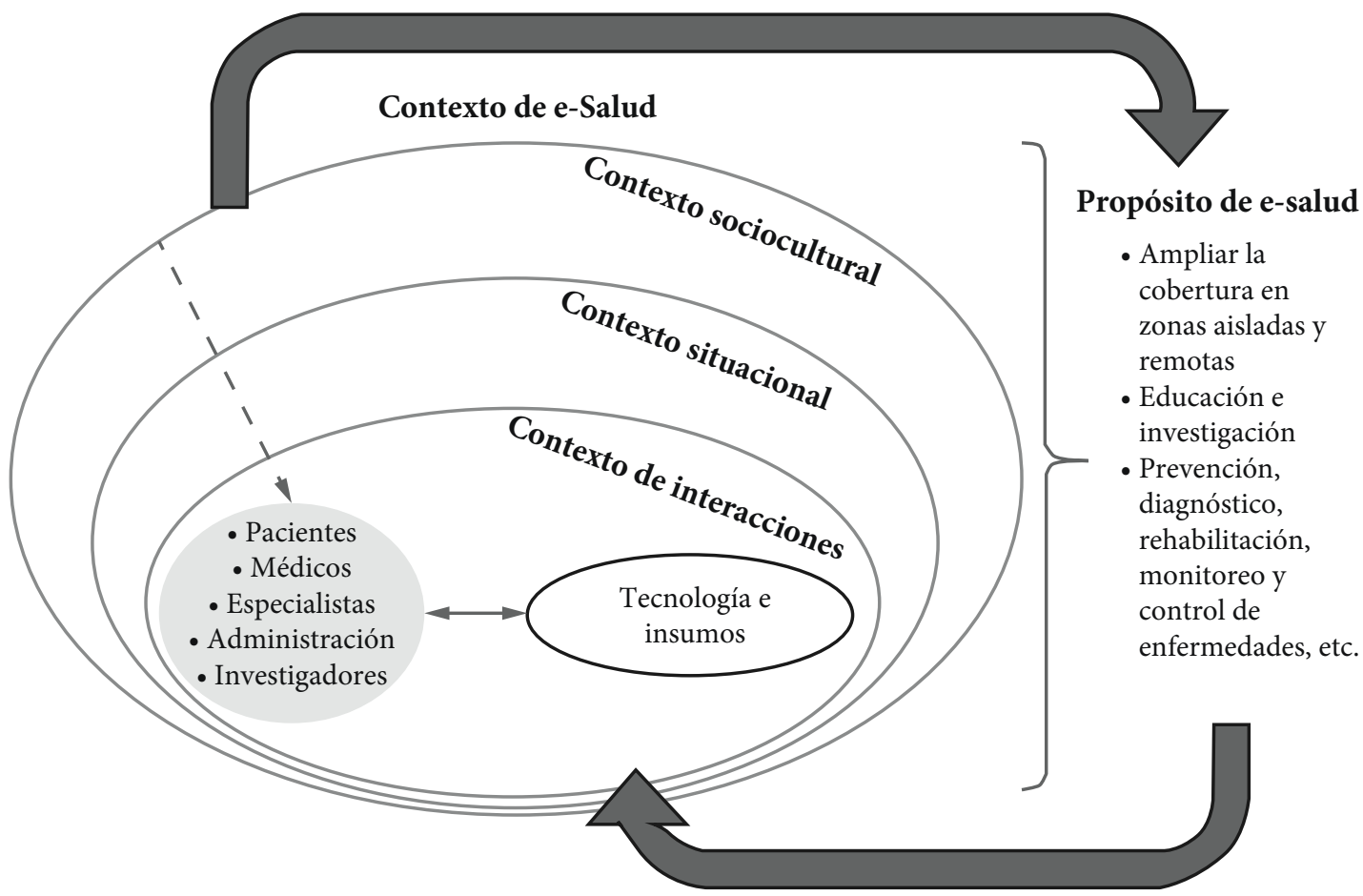

Figura 1. Componentes del ecosistema y el propósito de e-salud Fuente: elaboración propia

entonces una construcción con los siguientes elementos [16], tal como se muestra en la figura 2:

1. Generar una visión unificada sobre el problema de salud que se va a enfrentar, considerando la e-salud como una alternativa de solución (marco epistémico).

2. Llevar a cabo la lectura de la realidad dentro del contexto específico que se intervendrá. Se hace un análisis por parte de los participantes del programa de e-salud sobre la historicidad y la condición observada del contexto en donde se llevará a cabo la intervención. Se recomienda incluir en este análisis a agentes del contexto.

3. Plantear la condición deseada con base en la estrategia sugerida por Castañares-Maddox [12] sobre sistemas socioambientales, en la cual se identifican tres niveles de gestión en la toma de decisiones. Esta estrategia es un intento de actuar sobre la complejidad para lograr una conexión entre teoría y praxis, es decir, para implementar y gestionar los proyectos situados en el contexto específico. Los tres niveles son: a) estratégico; b) táctico, y c) operativo.

Estos tres niveles de organización, con actores interactuando inter e intraniveles, dan lugar a flujos de entrada y salida, los cuales determinan en sus interrelaciones la estructura del sistema complejo. En esta forma, en el desarrollo del programa, intervención o proyecto de e-salud, la toma de decisiones emerge de la interacción de los agentes en los tres niveles, lo cual daría lugar a acciones con mayor grado de coherencia e integración.

4. Planteamiento de preguntas conductoras, las cuales ayudarán a implementar y gestionar el proyecto para llegar a la condición deseada en función de lo analizado en la condición observada.

5. Desarrollar un plan de acción que dé respuesta a las preguntas conductoras.

6. Implementación, gestión y seguimiento. En esta dinámica, los procesos de retroalimentación 


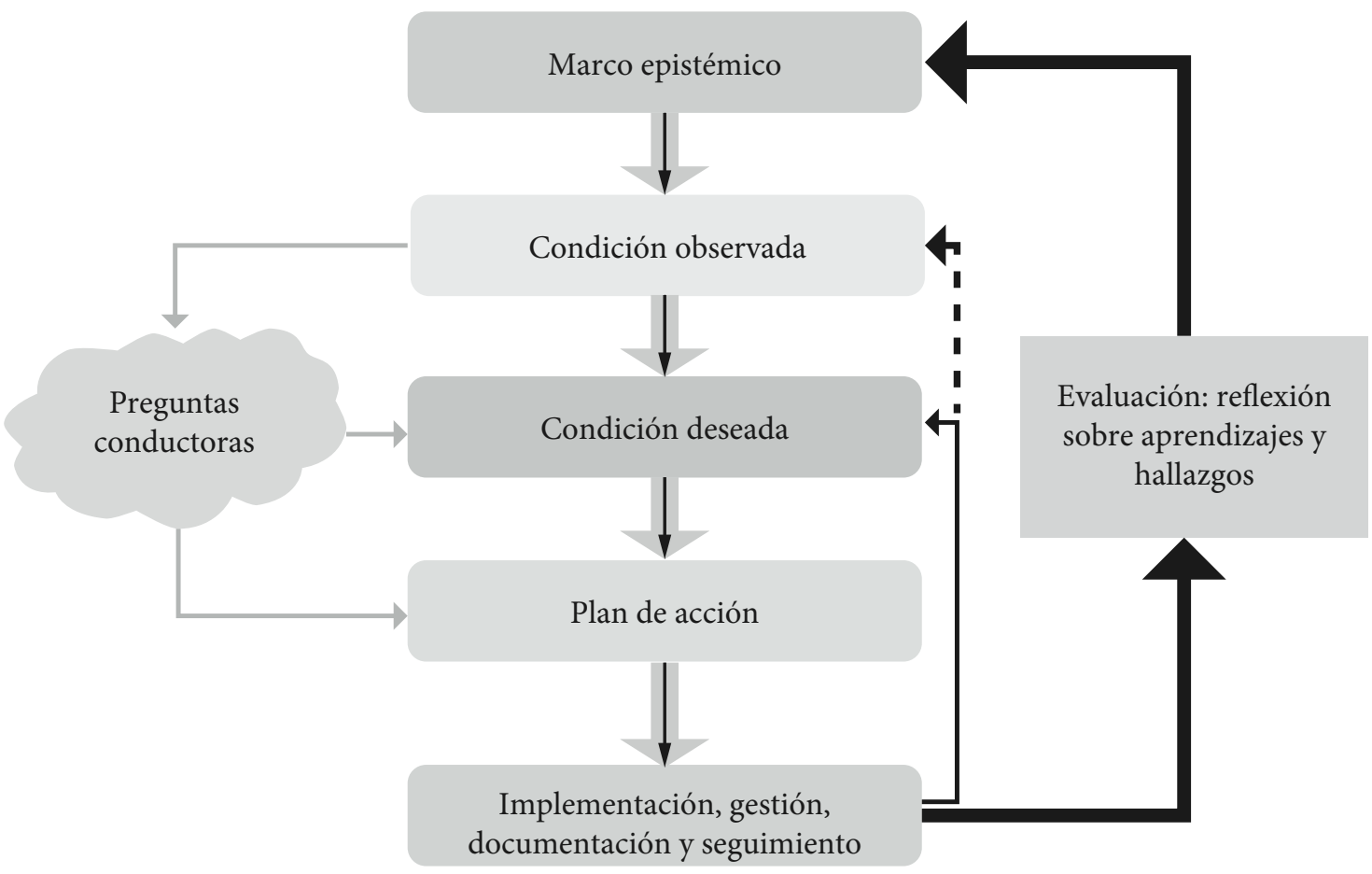

Figura 2. Proceso de construcción del espacio de estudio de la e-salud como un sistema complejo Fuente: elaboración propia

juegan un papel importante en la evaluación de la efectividad y pertinencia de proyectos; así se determina su ciclo de vida e impacto en el bienestar de la población intervenida. En el seguimiento es importante evaluar los logros y desafíos; además de reflexionar sobre aprendizajes y hallazgos que puedan aportar al planteamiento de etapas sucesivas de la intervención o nuevas intervenciones.

\section{Resultados: actuando sobre la complejidad. Recomendaciones para el diseño de políticas públicas en e-salud}

Se pueden identificar dos enfoques sobre la relación y uso de la complejidad en el contexto de las políticas públicas [17]. Una de las corrientes considera que los sistemas generadores de políticas públicas se comportan como sistemas complejos. La otra corriente enfatiza las características de los sistemas complejos sin necesariamente utilizar el lenguaje de la complejidad. En este artículo se usan elementos de ambas corrientes para el planteamiento de nuestra propuesta de análisis.

Aguilar Villanueva [18] considera que las políticas públicas consisten en el diseño de una acción colectiva intencional, el curso que efectivamente toma la acción como resultado de varias decisiones e interacciones y, en consecuencia, los hechos reales que la acción colectiva produce. Por esta razón, una política pública es una solución que se oferta partiendo de una condición social. El mismo autor [19] menciona que hay una diferencia entre las políticas gubernamentales y las públicas, en el sentido de que estas últimas no son decisión del actor gubernamental, sino que constituyen un curso de acción que se desata y efectivamente se desarrolla, intencional y reactivamente, por varios actores gubernamentales, por otros poderes estatales, actores políticos, grupos económicos u organizaciones sociales.

Las políticas públicas requieren del consenso social. Por lo tanto, para lograr su aceptación e 
implementación, necesitan ser planteadas y discutidas públicamente y principalmente ser convincentes de que solucionarán de manera viable la condición social tratada. Al estar de acuerdo con los planteamientos presentados para la solución de una determinada condición social, los actores pueden ser persuadidos para participar en la solución e implementación de la política pública. Un elemento importante a considerar es que, al presentar una política pública, se supone la presencia de los ciudadanos informados e interesados en el tema que se debatirá, a quienes se les deberá proporcionar espacios de participación ad hoc ideales para que tengan intervención en el proceso. Por esto, al hacer referencia al enfoque de los sistemas complejos puede decirse que gobernar de acuerdo a las políticas públicas significa gobernar incorporando la opinión y participación de los ciudadanos, que es el camino óptimo para generar las políticas públicas [20].

En cuanto al sector salud, la oms [21] afirma que:

En los programas nacionales de salud cerca de dos terceras partes de los países no disponen de una política nacional de tecnologías sanitarias que orienten la planificación, evaluación, adquisición y gestión de los dispositivos médicos. Por lo que la inexistencia de una política puede hacer que se invierta en dispositivos médicos que no se ajusten a las necesidades prioritarias de la población, que sean incompatibles con las infraestructuras y servicios existentes o cuyo mantenimiento sea demasiado caro. Este desperdicio puede minar los sistemas de salud en su conjunto, desviando fondos necesarios para otros servicios esenciales.

Al iniciar la actuación sobre la complejidad de acuerdo con lo descrito en la figura 2, es necesario definir los objetivos y alcances de una intervención concreta y un contexto particular. Las preguntas conductoras relacionan las necesidades del contexto con sus características e historicidad según lo sugerido en los pasos 1, 2 y 3 de la figura 2. Para tal efecto, se lleva a cabo el análisis de niveles propuesto en la figura 3, con lo cual obtendremos un corte de la condición observada del contexto en particular y así identificar las funciones y responsabilidades de los actores inter e intraniveles.

\section{Componentes analíticos de un sistema complejo}

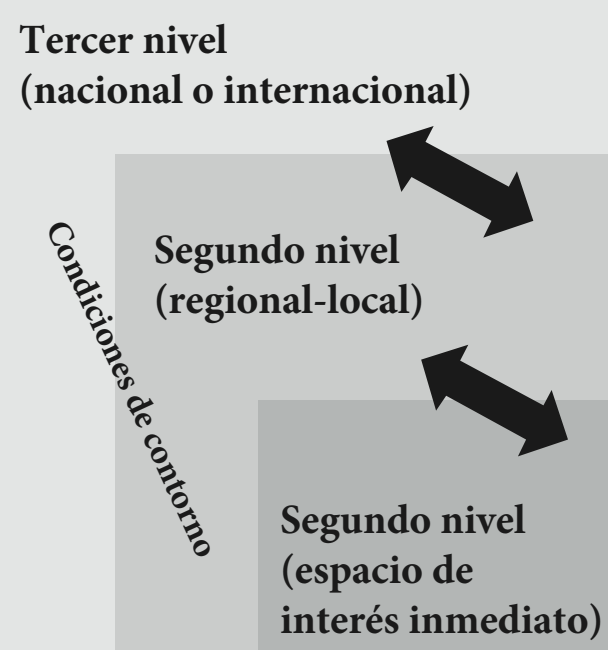

Niveles de decisión Caso de estudio: telediagnóstico

\begin{tabular}{|l|l|}
\hline $\begin{array}{l}\text { Estratégico } \\
\text { (define objetivos } \\
\text { globales a largo plazo) }\end{array}$ & $\begin{array}{c}\text { Organizaciones nacionales } \\
\text { e internacionales de } \\
\text { salud y tic }\end{array}$ \\
\hline $\begin{array}{l}\text { Táctico } \\
\text { Traduce la } \\
\text { estrategia al plano } \\
\text { regional-local }\end{array}$ & $\begin{array}{c}\text { Centros de salud } \\
\text { regionales y locales }\end{array}$ \\
\hline $\begin{array}{l}\text { Operativo } \\
\text { Participación } \\
\text { directa con los } \\
\text { objetos }\end{array}$ & $\begin{array}{c}\text { Actores de subsistema } \\
\text { humano, tecnológico } \\
\text { y económico }\end{array}$ \\
\hline
\end{tabular}

Figura 3. Marco de referencia: contextualización del espacio de estudio en los tres niveles del ámbito de la decisión Fuente: elaboración propia 
$\mathrm{Al}$ analizar las interacciones en el nivel operativo para un caso hipotético de telediagnóstico, se ve que existe una interacción entre médicos generales y especialistas a través de dispositivos médicos, diferentes tipos de software aplicados a videoconferencia y una red de acceso a Internet, por lo que para este caso particular la interacción que exista entre los médicos y la tecnología es importante, ya que va a ser la mediadora para que se pueda brindar un diagnóstico oportuno y va a ayudar a tomar una decisión para actuar respecto al paciente. En este aspecto, las habilidades digitales de los médicos y los aspectos de usabilidad y aceptación de la tecnología son muy importantes [22].

Siguiendo con el procedimiento propuesto, se debe llevar a cabo un análisis de las interacciones en el nivel táctico. Como se observa en la figura 3, este nivel va a mediar entre los niveles operativo y estratégico, por lo que juega un papel importante al momento de la creación de políticas públicas. En el caso del ejemplo de telediagnóstico, existen interacciones entre los centros de salud regionales que intervienen en la toma de decisiones que se llevarán a cabo en el nivel operativo. Se observa también que los costos de insumos y la infraestructura física están relacionados con cada uno de dichos centros de salud, ya que su administración depende de los recursos financieros asignados.

Finalmente, en el nivel estratégico, intervienen las instituciones con responsabilidades en los procesos de e-salud tanto en el ámbito nacional como en el internacional. El análisis de las interacciones inter e intranivel aporta información importante para llegar a la condición deseada y representa una lectura del funcionamiento del caso de telediagnóstico tomado como ejemplo. Con la información obtenida de esta lectura se podrán plantear las intervenciones de e-salud y, dependiendo de las preguntas conductoras, se apoya el proceso de conformación de las políticas públicas asociadas.

\section{Caso de estudio}

El marco de referencia propuesto se ha aplicado en diferentes intervenciones de e-salud en México; un caso específico es el relativo al planteamiento del proyecto de investigación "Teleepidemiología de

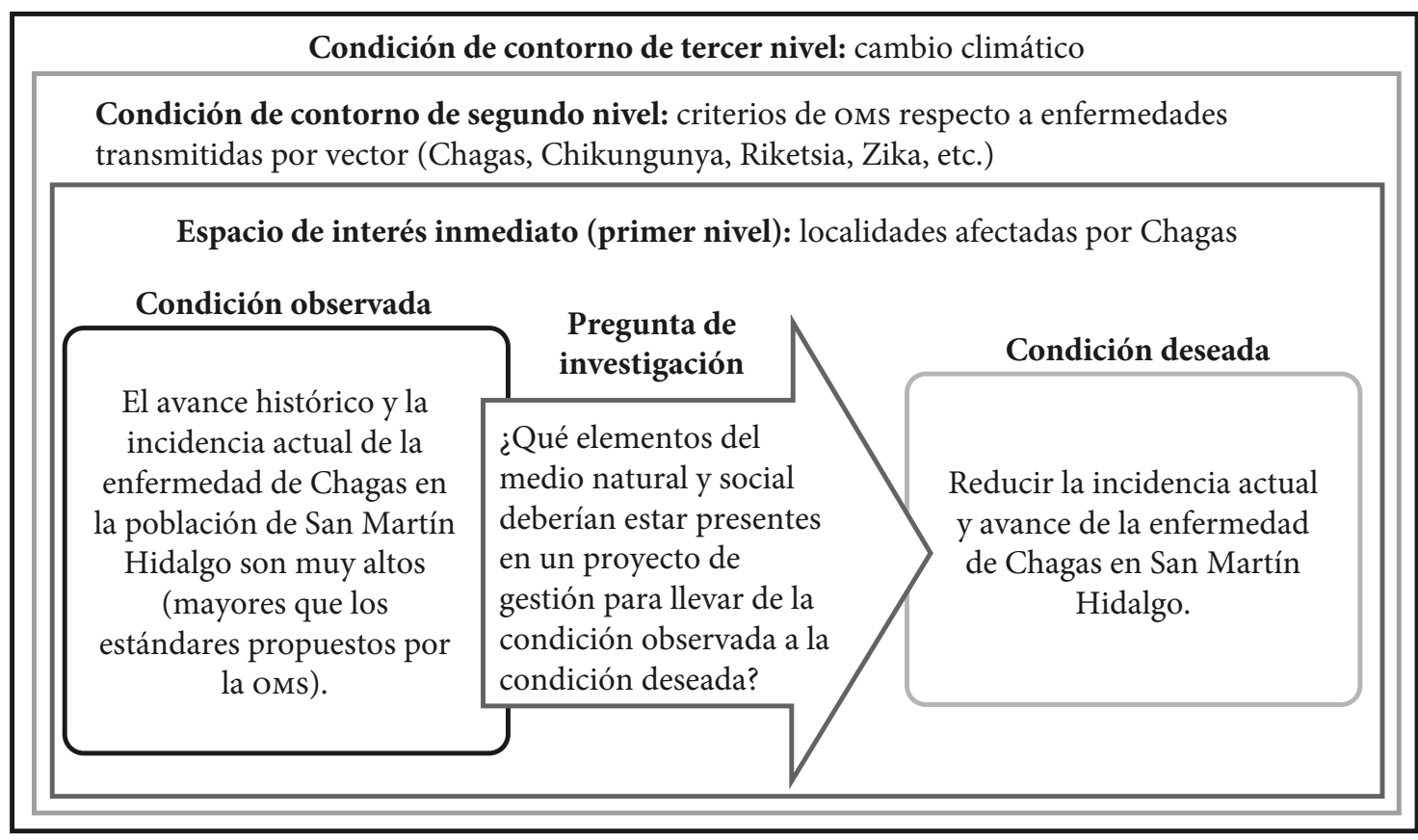

Figura 4. Marco conceptual y operativo para un modelo de gestión dirigido a la atención a zonas afectadas por la enfermedad de Chagas Fuente: elaboración propia 
enfermedades transmitidas por vectores", auspiciado por la Red de Ciencia y Tecnología Espacial que hasta el 2016 fue la Red Temática del Consejo Nacional de Ciencia y Tecnología de México [23]. El objetivo de este proyecto consistió en desarrollar una herramienta computacional que permita predecir brotes epidemiológicos mediante el uso e interpretación de tecnología espacial. Previo a la intervención específica, se llevó a cabo la lectura de la condición observada de San Martín Hidalgo en Jalisco, México, población que ha sido afectada durante varios años por la enfermedad de Chagas. El grupo de investigación adoptó el marco de referencia propuesto en este artículo, el cual, en forma resumida, se presenta en la figura 4 . Como puede observarse en dicha figura, la tecnología no es el elemento fundamental del proyecto. El aspecto esencial del proyecto es llegar a una condición en la cual se pueda reducir la incidencia y avance de la enfermedad de Chagas en la población objetivo. Las aplicaciones de la tecnología satelital, para el caso teleepidemiología, constituyen herramientas que permiten aproximarse al cumplimiento de los objetivos del proyecto, cuyo centro de atención es la salud de la población, a través de las preguntas de investigación.

El planteamiento llevado a cabo para el caso de Chagas se extrapolará al caso de Zika mediante la incorporación de investigadores brasileños, con el objeto de enriquecer el contexto de estudio y considerar aspectos de interdisciplinarios inmersos en los proyectos de teleepidemiología. Esta investigación ha seguido su curso durante el 2017, incorporando aspectos de georreferenciación, aplicaciones móviles y estudios socioambientales.

\section{Discusión}

Con el objeto de hacer operativa nuestra propuesta, la generación de un plan de acción, como se propuso en la figura 2, se convierte en un elemento clave en la gestión de las intervenciones. Este plan de acción se encuentra en constante observación por los agentes de los niveles tácticos y estratégico, para, por un lado, diseñar nuevas políticas públicas o, por otro lado, fortalecer las existentes. Debido a la naturaleza adaptiva y dinámica del ecosistema de e-salud, donde el ejemplo hipotético de telediagnóstico se lleva a cabo, es necesario considerar procesos de retroalimentación entre los diferentes niveles para optimizar y actualizar periódicamente las políticas públicas asociadas. Un posible análisis puede llevarse a cabo mediante el uso de modelos basados en agentes (ABM, por sus siglas en inglés) y complementarlo con otros modelos complementarios que emplean redes neuronales; con estos se pueden generar diferentes escenarios que permitan llevar a cabo un diseño adecuado y apoyar la toma de decisiones de la intervención de e-salud, como lo describió Rojas-Mendizábal [24]. En este artículo, la autora enfatiza que el propósito de los desarrollos sociotécnicos debería ser el beneficio directo del usuario final, y considera las comparaciones costo-beneficio y costo-efectividad en la evaluación de las intervenciones de e-salud. Siguiendo la misma línea, el marco de referencia propuesto puede servir como plataforma para el desarrollo de estándares con participación local, lo cual resulta imprescindible para reducir las brechas de innovación entre los países en vías de desarrollo y los más avanzados.

Desde su origen, la e-salud ha sido afectada por los elementos socioculturales y, aunque este hecho no haya sido suficientemente reconocido, o cuando menos observado, estos elementos deben ser incluidos en los programas educativos sobre e-salud.

La e-salud es un campo abierto de investigación y desarrollo tecnológico. Bajo estas circunstancias, se propone como trabajo futuro la posibilidad de integrar un enfoque epistemológico sólido para cada programa, intervención o proyecto de e-salud capaz de interpretar y explicar la interacción entre los sistemas sociales y tecnológicos. Se reconoce que la complejidad inherente de los sistemas sociotécnicos, particularmente en las conversaciones entre teoría y praxis, debe ser analizada con mayor profundidad y explorar otras alternativas complementarias, las cuales puedan coadyuvar al diseño de proyectos (inclusión digital en el sector salud, redes y dispositivos médicos de nueva generación y otros) de manera holística y contribuir de esta forma a su aplicación al bienestar social; sobre todo poder enfrentar los retos del cambio tecnológico abrumador que actualmente la sociedad experimenta. Una alternativa de análisis en proceso de investigación es el vínculo de conceptos de Weaver [25] sobre complejidad organizada, y la propuesta de Functowicz y Ravetz sobre ciencia postnormal [26]. En esta investigación se espera encontrar nuevos elementos que solidifiquen nuestro marco 
metodológico para proponer procesos de gestión que incorporen factores que surgen en la interacción de sistemas complejos naturales y humanos, tales como riesgos, compromiso humano y valores; estos son clave para la toma de decisiones en la implementación práctica de los proyectos.

Nuestro propósito ha sido aplicar la perspectiva integral que ofrece la complejidad para conformar un marco de referencia que dé pie al planteamiento de políticas públicas en el contexto de e-salud, tomando en cuenta la participación de todos los actores involucrados, sus interacciones y el reconocimiento del contexto; esto resulta fundamental para que la e-salud sea un vehículo efectivo de solidaridad y desarrollo social.

\section{Conclusiones}

El marco conceptual ofrecido sobre la concepción de e-salud sugiere una conexión que involucra varios sistemas interrelacionados (cognitivos, tecnológicos, lingüísticos, socioculturales y otros); admite, por lo tanto, un enfoque interdisciplinario a manera de "puente conceptual" entre disciplinas que pudieran, en una primera instancia, observarse como incompatibles. Una excesiva fragmentación y la falta de una visión clara sobre el servicio de la e-salud a la sociedad no permiten abordar una realidad en la que participan dispositivos, equipos, procesos, programas, y lo más importante, seres humanos.

El acelerado cambio tecnológico y la convergencia digital están creando brechas de conocimiento e innovación cada vez más difíciles de reducir y enfrentar; esta condición convierte a la e-salud en una oportunidad y en un desafío. En este escenario surgen preguntas que tendrán que abordarse de manera urgente para aprovechar el potencial de las TIC en forma equitativa e integral: ¿cuáles son las aplicaciones y contextos donde la e-salud es pertinente para contribuir al desarrollo? ¿Qué elementos regulatorios y normativos son necesarios para asegurar que la e-salud sea de beneficio a la población, y no solo elemento de consumismo? ¿Qué estrategias son requeridas para reducir la abismal brecha de innovación en e-salud entre países desarrollados y el resto del mundo? ¿qué estrategias de formación de capital humano son necesarias para aprovechar los avances de la e-salud en el progreso y bienestar social? Tal vez sea necesario detenerse a reflexionar sobre las implicaciones del trepidante y absorbente cambio tecnológico, e identificar los límites de la digitalización de cara a privilegiar lo fundamentalmente humano.

\section{Referencias}

[1] OMs, "Trade, Foreign Policy, Diplomacy and Health", World Health Organization, 2014.

[2] P. Sharp, P y S. Hockfield, "Convergence: The Future of Health", Science, vol. 355, n. ${ }^{\circ}$ 6325, p. 589, Feb. 2017. [Online]. doi: https://doi.org/10.1126/science. aam8563.

[3] R. A. Anderson, B. F. Crabtree y R. R. Jr. McDaniel, "Case Study Research: The View From Complexity Science," Qualitative Health Research, vol. 15, n. ${ }^{\circ}$ 5, pp. 669-85, mayo 2005. [Online]. doi: https://doi. org/10.1177/1049732305275208

[4] N. Almeida-Filho, "Complejidad y Transdisciplinariedad en el Campo de la Salud Colectiva: Evaluación de Conceptos y Aplicaciones", Salud Colectiva, vol. 2, n. ${ }^{\circ}$ 2, pp. 123-46, mayo 2016. [Online]. doi: https://doi.org/10.18294/sc.2006.61

[5] R. M. Raims Andalia y P. L. Sotolongo Codina, "Aportes del pensamiento y las ciencias de la Complejidad al estudio de los determinantes de la salud", Revista Cubana de Salud Pública, vol. 35, n. ${ }^{\circ}$ 4, pp. 65-77, Dic. 2009. [Online]. Disponible en http:// scielo.sld.cu/scielo.php?script=sci_arttext\&pid $=$ S0864-34662009000400008

[6] Organization for Economic Cooperation and Development (OECD), "Applications of Complexity Science for Public Policy: New Tools for Finding Unanticipated Consequences and Unrealized Opportunities," OCDE Global Science Forum, Sept. 2009. [Online]. Disponible en https://www.oecd. org/science/sci-tech/43891980.pdf

[7] ETSI, "Quality of Experience (QoE) Requirements for Real-time Communication Services," European Telecommunications Standards Institute, Francia, Reporte Técnico V1.0.2 (2010-01), 2010.

[8] A. Martínez, Bases metodológicas para evaluar la viabilidad y el impacto de proyectos de telemedicina, Washington, D.C.: Organización Panamericana de la Salud, Organización Mundial de la Salud, 2001.

[9] E. Morin y M. Pakman, Introducción al pensamiento complejo. Barcelona: Gedisa, 1994.

[10] Y.-Y. Liu, J. J. Slotine, y A.-L. Barabasi, "Observability of Complex Systems," Proceedings of the National Academy of Sciences, vol. 110, n. ${ }^{\circ}$ 7, pp. 2460-5, 
Ene. 2013. [Online]. doi: https://doi.org/10.1073/ pnas. 1215508110

[11] R. Axelrod y M. D. Cohen, Harnessing Complexity: Organizational Implications of a Scientific Frontier. Nueva York: Basic Books, 2000.

[12] E. J. Castañares-Maddox, Sistemas complejos y gestión ambiental: el caso de corredor biológico mesoamericano. México: Comisión Nacional para el Conocimiento y Uso de la Biodiversidad, 2009.

[13] R. García, Sistemas complejos: conceptos, métodos y fundamentación epistemológica de la investigación interdisciplinaria. Barcelona: Gedisa, 2006.

[14] Congreso de Colombia, "Ley N. ${ }^{\circ} 1.419$, Lineamientos para el desarrollo de la telesalud en Colombia”, 2010.

[15] J. Thompson Klein, "Interdisciplinarity and Complexity: An Evolving Relationship," E-CO Special Double Issue, vol. 6, n. ${ }^{\circ}$ 1-2, pp. 2-10, 2004.

[16] J. M. Aguilar de la Peña, Pobreza y marginación en la mixteca poblana: un análisis desde el enfoque de sistemas complejos, B. Ortiz Espejel y G. Duval, Eds. Puebla: Universidad Iberoamericana Puebla: Colegio de Posgraduados, 2008.

[17] P. Cairney, "Complexity Theory in Political Science and Public Policy: Complexity Theory", Political Studies Review, vol. 10, n. ${ }^{\circ}$ 3, pp. 346-58, Sept. 2012. [Online]. doi: https://doi.org/10.1111/j.1478-9302.2012.00270.x
[18] L. F. Aguilar, La implementación de las politicas. México: Miguel Ángel Porrúa, 1996.

[19] L. F. Aguilar, La hechura de las políticas. México: Miguel Ángel Porrúa, 1996.

[20] E. Lahera, Introducción a las políticas públicas. Santiago: Fondo de Cultura Económica, 2002.

[21] World Health Organization, "E-Health," who.

[22] V. Venkatesh y X. Zhang, "Unified Theory of Acceptance and Use of Technology: U.s. Vs. China", Journal of Global Information Technology Management, vol. 13, n. ${ }^{\circ} 1$, pp. 5-27, Ene. 2010. [Online]. doi: https://doi.org/10.1080/1097198X.2010.10856507

[23] "Red de Ciencia y Tecnología del Espacio." Proyectos 2016, Proyecto 10. [Online]. Disponible en http:// www.redcyte.org.mx/proyectos_2016.html.

[24] V. A. Rojas-Mendizábal, A. Serrano-Santoyo, R. Conte-Galván, y A. Gómez-González, "Toward a Model for Quality of Experience and Quality of Service in e-health Ecosystems," Procedia Technology, vol. 9, pp. 968-74, 2013. [Online]. doi: https://doi. org/10.1016/j.protcy.2013.12.108

[25] W. Weaver, "Science and Complexity", Facets of Systems Science, Boston, MA: Springer us, 1991, pp. 449-56.

[26] S. Funtowicz and J. R. Ravetz, "Post-normal Science", International Society for Ecological Economics, 2003. [Online] Disponible en http://isecoeco.org/ pdf/pstnormsc.pdf 